\title{
GOBIERNO ABIERTO: TRANSPARENCIA Y ACCESO A LA INFORMACIÓN EN LA ADMINISTRACIÓN DE LOS RECURSOS MATERIALES
}

Dalia Navarro Olea ${ }^{1}$

SUMARIO: Introducción II.Desarrollo.III Conclusiones y Recomendaciones

RESUMEN

El objetivo de este documento es realizar un análisis bibliográfico de resultados derivados de la implementación de los lineamientos normativos establecidos en materia de transparencia y acceso de información relacionados con la Administración de los Recursos Materiales.

Palabas Clave: Administración de recursos materiales,Trransparencia,Gobierno abierto

\section{OPEN GOVERNMENT: TRANSPARENCY AND ACCESS TO INFORMATION IN THE ADMINISTRATION OF MATERIAL RESOURCES}

\author{
Dalia Navarro Olea ${ }^{2}$
}

SUMMARY: Introduction II. Development. III Conclusions and Recommendations

\begin{abstract}
In this document the objective is to carry out a bibliographic analysis of the results derived from the implementation of the normative guidelines established in the matter of transparency and access to information related to the Management of Material Resources.
\end{abstract}

Key Words: Management of material resources, Transparency, Open government

\footnotetext{
${ }^{1}$ Maestrante del Programa Maestria en Administracion Pública del ISAPAC. Correo electrónico:dnavarro_o@hotmail.com

${ }^{2}$ Maestrante del Programa Maestria en Administracion Pública del ISAPAC. Correo electrónico:dnavarro_o@hotmail.com
} 


\section{Gobierno abierto: Transparencia y acceso a la información en la administración de los recursos materiales}

Volumen 12, Número 22 ene-jun 2020

\section{INTRODUCCIÓN}

Las Dependencias y Entidades de la Administración Pública están sujetas a un nuevo modelo cuyo propósito fundamental es la mejora de la gestión, la reducción del gasto, la adopción de mejores prácticas administrativas y el establecimiento de un esquema de control interno institucional que optimice la función y las actividades de las unidades administrativas del Sector Publico.

Desde el enfoque de Gobierno Abierto, enfrentamos el reto de mejorar los niveles de transparencia y acceso a la información derivada de la administración de los recursos materiales, mediante la apertura de datos públicos, facilitando la participación ciudadana y favoreciendo la generación de espacios de colaboración entre servidores públicos, la sociedad civil y el sector privado.

Acorde a Kisnerman "los recursos materiales, son aquellos que constituyen la infraestructura y los equipamientos básicos del Estado y que son orientados a satisfacer los fines últimos de la sociedad. ${ }^{3}$

La administración de los recursos materiales bajo esta perspectiva, implica el uso eficiente y racional de estos recursos, a fin de que su destino obedezca a los requerimientos y fines de la institución para el logro de una mayor eficacia, eficiencia y congruencia en su funcionamiento. ${ }^{4}$

De lo anterior se desprende la necesidad de que la administración de los recursos públicos y en particular los materiales, se conduzcan bajo criterios de honestidad, eficacia y transparencia, en provecho de la sociedad.

Desde el enfoque de Gobierno Abierto, enfrentamos el reto de mejorar los niveles de transparencia y acceso a la información derivada de la administración de los recursos materiales, mediante la apertura de datos públicos, facilitando la participación ciudadana y favoreciendo la generación de espacios de colaboración entre servidores públicos, la sociedad civil y el sector privado.

\footnotetext{
Vide: Enriquez Rubio Ernesto. Administración de recursos materiales en el sector público:Enfoque introductorio.pdf, pp.77 [en línea]Disponible en https://www.inap.mx/portal/images/pdf/book/14452.pdf ${ }^{4}$ Ibidem p.79
} 
La información sobre las decisiones y el quehacer gubernamental debe ser abierta, completa, oportuna, gratuita y de fácil acceso para el público. Ello supone que los datos públicos deben cumplir con parámetros y estándares comúnmente reconocidos, como: estar disponibles en un formato bruto, ser susceptibles de que se los procese, ser accesibles a través de herramientas tecnológicas y de comunicación.

El objetivo planteado es realizar un análisis bibliográfico de resultados derivados de la implementación de los lineamientos normativos establecidos en materia de transparencia y acceso de información relacionados con la Administración de los Recursos Materiales.

Para ello, analizamos el contenido de diversas fuentes bibliográficas identificando tanto la obligatoriedad establecida por la Ley Federal de transparencia y Acceso a la Información Pública, así como por la Ley de Acceso a la Información Pública del Estado de Sonora y demás ordenamientos relacionados.

De igual manera, consideramos aspectos de la experiencia en otros países en relación a este tema, así como la implementación de la estrategia de Gobierno Abierto y las dificultades encontradas tanto por parte de la propia Administración Pública como por la ciudadanía.

\section{DESARROLLO}

Las compras gubernamentales constituyen en el mundo uno de los renglones de gasto de mayor envergadura. Basta mencionar que, de acuerdo con estimaciones de la Organización Mundial de Comercio (OMC), las compras gubernamentales ascienden a un billón de dólares al año y en algunos estados de la comunidad internacional llegan a constituir más del diez por ciento del producto interno bruto. ${ }^{5}$

Recientemente y derivado de la proporción del gasto que implica la administración de los recursos materiales, se ha dado mayor importancia a las áreas de compras, implicando una mayor profesionalización en quienes realizan la

\footnotetext{
${ }^{5}$ Enriquez, R. E. (s.f.) op.cit.,p. 80
} 


\section{Gobierno abierto: Transparencia y acceso a la información en la administración de los recursos materiales}

Volumen 12, Número 22 ene-jun 2020

Dalia Navarro Olea

función, con mayor conocimiento de Leyes, Normas y Reglamentos, así como énfasis en aspectos éticos que garanticen, o por lo menos promuevan la transparencia y legalidad en la implementación de los procesos administrativos, así como el combate a la corrupción.

En otro sentido "los recursos materiales pueden ser definidos como el patrimonio de la institución y están constituidos por los bienes muebles, inmuebles y de consumo que hacen factible la operación de la misma. El elemento de apoyo para la consecución de las metas y objetivos de la administración pública"6 Considerando que el gasto invertido en el patrimonio, así como los bienes de consumo que permiten el funcionamiento de las Instituciones es de naturaleza pública y está orientada a satisfacer las demandas colectivas de la sociedad, es de suma importancia otorgar a la ciudadanía la información que permita evaluar los resultados de su administración y así poder determinar el éxito en el cumplimiento de los resultados por parte de los beneficiarios de dicha gestión.

En el marco de la evolución de la Teoría de la División de Poderes de Montesquieu a la Teoría de la división de Funciones, contemplada en nuestra Carta Magna, corresponde formalmente al Titular del Poder Ejecutivo ${ }^{7}$ conducir la administración pública, la cual es responsable de la adquisición, registro, almacenamiento y suministro de los bienes que requieren sus órganos y más aún de las previsiones de obras y servicios a la población.

Bajo esta premisa, es preciso que los recursos públicos se administren con eficiencia, eficacia y honradez, por lo que los gobiernos están obligados a ser cuidadosos de los recursos que tienen bajo su responsabilidad.

En este sentido como menciona Garibay "En el apartado de las compras gubernamentales, las demandas sociales constituyen uno de los renglones de gasto

\footnotetext{
${ }^{6}$ Enriquez Rubio Ernesto. Administración de recursos materiales en el sector público:Enfoque introductorio.pdf p.79 [en línea]Disponible en : https://www.inap.mx/portal/images/pdf/book/14452.pdf

7 Los otros Poderes (Legislativo, Judicial y Órganos Autónomos) materialmente realizan la función administrativa y son responsables de la adquisición, registro, almacenamiento y suministro de los bienes que requieren sus órganos debiendo también observar que los recursos públicos se administren con eficiencia, eficacia y honradez, por lo que tambien les obliga a ser cuidadosos de los recursos que tienen bajo su responsabilidad.
} 
público de mayor compromiso y es por eso que debe haber un uso correcto de los recursos que administran los servidores públicos". ${ }^{8}$

Sin embargo, es en este campo, en el de comprar y contratar, donde se presenta mayor vulnerabilidad para el favoritismo y la corrupción, es aquí en donde la transparencia y el acceso a la información constituyen una herramienta fundamental para la prevención y el control de la corrupción, así como un recurso invaluable para recuperar la credibilidad en el servidor público y promover la participación ciudadana en la implementación de nuevos proyectos de inversión y políticas públicas.

Como menciona Enríquez "El entorno internacional pone de manifiesto cómo los recursos públicos son cada vez más escasos y las necesidades sociales crecientes. La mayoría de los gobiernos enfrentan como hecho común, el cuestionamiento de una ciudadanía que demanda cada día, más y mejores resultados, así como una mayor transparencia en el manejo de los recursos públicos". 9

La transparencia y el combate a la corrupción son temas que en forma gradual han adquirido mayor importancia, en todos los niveles de Gobierno nacional e internacional.

En palabras de Enríquez "en consonancia con esto, la administración de Vicente Fox, hizo del combate a la corrupción y la construcción de la cultura de transparencia un pilar de su oferta de cambio."

El ex presidente Vicente Fox Quesada, inició su Administración con la creación de la Comisión Intersecretarial para la Transparencia y el Combate a la Corrupción en la Administración Pública Federal por sus siglas CITCC.

La CITCC fue instituida mediante Acuerdo presidencial publicado en el Diario Oficial de la Federación el 4 de diciembre del 2000. Según afirma Barrio Terrazas

\footnotetext{
8 Vide : Garibay Escobar, Sergio, Debe tener la administración pública una gestión eficiente de los recursos, Noticias Universidad de Sonora,25 de mayo de 2016 [en linea] disponible en: http://www.unison.edu.mx/noticias/default.php?id=21806

${ }^{9}$ Enríquez, op.cit.,p.99
} 


\section{Gobierno abierto: Transparencia y acceso a la información en la administración de los recursos materiales}

Volumen 12, Número 22 ene-jun 2020

Dalia Navarro Olea

"En este acto, se partió del principio de que el combate a la corrupción y la mejora de la transparencia de la gestión gubernamental son asuntos prioritarios y que forman parte de las agendas de todos aquellos que participan en la función pública.

Esta Comisión busca abatir los niveles de corrupción en el país y dar absoluta transparencia a la gestión y al desempeño de las instituciones y los servidores públicos de la administración pública federal, regulando y orientando los esfuerzos y acciones de las distintas dependencias y entidades de la administración pública para obtener mejores resultados y no compromisos débiles y cumplimientos parciales."10

El concepto de "transparencia" denota deber del gobierno de exponer y someter a escrutinio público la información relativa a su gestión, al manejo de los recursos que la Sociedad le ha confiado, a los criterios que sustentan sus decisiones, y a la conducta de sus funcionarios. ${ }^{11}$

Sin embargo, en la práctica el enfoque de la transparencia en un inicio ha estado enfocado al cumplimiento de la legalidad, partiendo del punto de vista de los diferentes órganos fiscalizadores y no de las necesidades reales de la ciudadanía la cual requiere por un lado la certeza de que los recursos públicos están siendo administrados de forma eficiente y honesta y por otro que la información que se le proporciona es clara y concisa para proporcionarle elementos de evaluación y no sólo constatar que se cumple desde el punto de vista normativo.

Es importante señalar que como lo mencionara la Secretaría de la Contraloría y Desarrollo Administrativo SECODAM, hoy Secretaría de la Función Pública, y el Instituto Nacional de Administración Pública INAP, la concepción de transparencia debe ser impulsada "desde fuera hacia adentro", desde la Sociedad

\footnotetext{
10Vide: Informe de Francisco Barrio Terrazas Presidente de la Comisión Intersecretarial para la Transparencia y el Combate a la Corrupción,2000 [en línea]Disponible en: www.programaanticorrupcion.gob.mx

11 Vide: CLAD Gobierno abierto y transparencia focalizada, en Tendencias y desafíos para América Latina y el Caribe,Nicolás Dassen y Juan Cruz Vieyra Editores Noviembre 2012 / Código de publicaciones: IDB-MG-137[en línea]Disponible en: http://siare.clad.org/fulltext/2231500.pdf
} 
hacia la Administración, acorde a la relación de Servidor Público y Sociedad, en la que el origen es el administrar los recursos para el beneficio social. ${ }^{12}$

Donde lo esencial es que la Sociedad pueda verificar a través de evidencias, que la Administración avanza en la implantación de mecanismos eficaces para forzar un desempeño transparente de sus funciones y agentes por encima e independientemente de la calidad ética de los mismos.

El campo de la Administración de los Recursos Materiales es un área de gran importancia y un asunto de interés general en lo que a requerimientos de información de la ciudadanía se refiere, especialmente vinculado a la rendición de cuentas del control del gasto.

Considerando que la Sociedad desconfía de la calidad moral de los Servidores Públicos, así como de la utilización de recursos de transparencia y acceso a la información que se presentan en forma confusa, parcial o sesgada, es de esperar que de ello se genere más descontento y resistencia de la ciudadanía a mostrar credibilidad en las Instituciones.

Es en este punto donde el concepto de Gobierno Abierto pretende facilitar los mecanismos para establecer una comunicación permanente y transparente entre la Administración y los ciudadanos, mediante la generación de espacios de participación con información clara, que permita integrar a la ciudadanía a la acción pública y simultáneamente reforzar el reclutamiento, la selección y la capacitación del recurso humano que garantice un adecuado manejo de las finanzas y una mayor disposición para la transparencia.

Como refiere Marcela Rastrepo "Hoy en día se reconoce que el acceso a la información pública no sólo afecta positivamente la agenda anticorrupción sino la agenda de la calidad de la democracia y el buen gobierno. El éxito en la gestión pública en relación con los sistemas de compras y adquisiciones, los sistemas de planificación, evaluación y control interno, la gestión del talento humano, la promoción de la participación ciudadana, la rendición de cuentas y el acceso de los

12 Vease Enríquez Rubio Ernesto,op.cit. pp. 101 y ss 


\section{Gobierno abierto: Transparencia y acceso a la información en la administración de los recursos materiales}

Volumen 12, Número 22 ene-jun 2020

Dalia Navarro Olea

ciudadanos a los bienes y servicios del Estado muestra no sólo capacidades eficientes de ejecución sino capacidades éticas y de integridad pública". ${ }^{13}$

Acorde a lo señalado por Enriquez "es importante destacar que la implementación de una auténtica transparencia requiere la introducción no sólo de ofrecer acceso a ciertas colecciones o catálogos de datos diseñados y sesgados, debe proporcionar información relevante, oportuna, pertinente y confiable por parte de quienes administran los recursos materiales e incluso considerar sanciones en las organizaciones públicas, privadas y sociales, para promover una cultura de la integridad."

Es necesario establecer los mecanismos de control y lucha contra la corrupción y de ampliar la transparencia del Estado, tanto por los funcionarios de la administración pública como para la sociedad civil de modo general, permitiendo el monitoreo sistemático de los gastos públicos para la identificación de situaciones que puedan constituir irregularidades, a fin de evitarlas antes de que ocurran.

La incorporación de tecnología informática constituyó una condición indispensable para el soporte y el procesamiento transparente del volumen de información que tramitan las Dependencias y Organismos, situación que ha requerido la capacitación del recurso humano así como la implementación de sistemas que permitan estructurar la información de manera clara y concisa.

Considerando que en el ámbito de actuación de quienes administran los recursos materiales existe la posibilidad de presentar información, transparente, translúcida y opaca, la idea de inducir a través de dichas tecnologías, una creciente ampliación de los espacios transparentes y translúcidos, reduciendo cada vez más los opacos a aquellos aspectos que por su naturaleza requieran el calificativo de confidenciales.

En este punto cabe recordar que como bien lo indica el Instituto Nacional de Administración Pública "la factibilidad de esta estrategia de transparencia está en

\footnotetext{
${ }^{13}$ Restrepo Hung Marcela, "Promoción de la transparencia focalizada en la gestión del acceso a la vivienda en Colombia: el caso del programaTACS",2012[en línea]disponible en: http://bibliotecadigital.tamaulipas.gob.mx/archivos/descargas/827d5b7339e1d882b2fdd009d5c7a8d 0c8617096.pdf
} 
función de la voluntad política de implantar sistemas que permitan romper los nichos de opacidad que aún subsisten en la Administración."La experiencia de la implementación de Gobierno abierto en otros países, particularmente en lo que respecta a la transparencia en el control del gasto, deja clara la preocupación que surge a partir de sospechas y denuncias relativas al comportamiento de los hombres públicos, percibiéndose la necesidad de disponer de instrumentos eficaces para inducir conductas más responsables, transparentes y honestas, así como para prevenir, disuadir y sancionar las eventuales conductas indebidas.

Otro aspecto importante a considerar es quiénes son los usuarios de la información, los cuales suelen ser ciudadanos, pero también pueden ser empresas, los jueces, los organismos de control y hasta los mismos funcionarios públicos, no participan sólo por haber sido invitados por un gobierno a hacerlo, para ello debe haber una oportunidad que no depende únicamente de la existencia de canales facilitados por el Estado, la necesidad se presenta generalmente cuando un sector de la población se ve amenazado por una política que pone en riesgo su situación actual.

Los ciudadanos no son por naturaleza participantes activos, para hacerlo deben tener una causa o razón que los motive y esto generalmente está relacionado a algún interés económico, un valor profundamente arraigado o un derecho legítimo que ha sido amenazado por el Estado.

En la medida que sea logrado el objetivo de Gobierno Abierto en su promoción de la participación ciudadana y se generen cambios culturales significativos en la forma de pensar, se logrará el empoderamiento de la sociedad en lo que por derecho le corresponde, vigilar el correcto manejo de los recursos públicos y participar activamente en la formulación de la políticas públicas.

A continuación, describimos las experiencias en materia de transparencia en varios países de Latinoamérica, que si bien no son todas relacionadas a la Administración de los Recursos Materiales, proporcionan resultados adquiridos bajo metodologías científicas rigurosas que pueden extrapolarse a nuestra materia.

En Colombia, Marcela Restrepo expone "el principio de la máxima publicidad en los asuntos públicos como uno de los pilares de la transparencia y la lucha contra 


\section{Gobierno abierto: Transparencia y acceso a la información en la administración de los recursos materiales}

Volumen 12, Número 22 ene-jun 2020

Dalia Navarro Olea

la corrupción, menciona que una mejor y más abundante información pública contribuye con la participación ciudadana. La información permite a las instituciones y actores sociales reducir el margen de incertidumbre y discrecionalidad, favoreciendo la eficiencia y eficacia en la gestión de bienes y servicios públicos y reduciendo los espacios de oportunidad para las prácticas corruptas. ${ }^{14}$

En la misma línea cabe citar la experiencia de la Contraloría General de Brasil, que con el fin de perfeccionar los mecanismos de control y lucha contra la corrupción y de ampliar la transparencia del Estado, estableció en 2008 el Observatorio del Gasto Público, el cual permite realizar un monitoreo sistemático de los gastos públicos para la identificación de situaciones que puedan constituir irregularidades, a fin de evitarlas antes de que ocurran. ${ }^{15}$

Esta herramienta ha demostrado no sólo ser eficaz por su capacidad de identificar indicios de irregularidades, sino también por su influencia positiva para los funcionarios de la administración pública haciendo ver que la utilización de la tecnología hizo que fuera auditable lo que antes habría sido imperceptible a los ojos de los auditores.

Con base al desglose de planes de acción de la Open Government Partnership Honduras presentó iniciativas en los planes de acción nacional relacionados a publicación de información sobre finanzas públicas (presupuesto abierto). Estrategia de empoderamiento ciudadano sobre información presupuestaria, convenios marco y adquisiciones públicas y Profundización de la

\footnotetext{
14 Restrepo Hung Marcela, op.cit.,p s/n

15 Vide: Mecanismo de seguimiento de la OEA/Ser.L. Implementación de la Convención SG/MESICIC/doc.330/12 rev. 4 Interamericana contra la Corrupción 14 Septiembre 2012 Vigésima Reunión del Comité de Expertos :Observatorio del Gasto Público (ODP)213": esta iniciativa, construida sobre los pilares de la capacitación humana, la aplicación de una metodología científica, y apoyada en la tecnología de la información, tiene capacidad de producir información y conocimientos para contribuir a realizar y acelerar la labor de los administradores en la toma de decisiones estratégicas por medio del seguimiento del gasto público utilizando en forma innovadora el cruzamiento de un gran volumen de datos de las más variadas fuentes, que contribuyen tanto a la finalidad de apoyar el mejoramiento de la gestión pública en cuanto a aumento de la eficiencia de los programas de gobierno, los procedimientos y los gastos, como a identificar situaciones de riesgo o de violación de la integridad de procesos y servicios.2012 [en línea]Disponible en:http://www.oas.org/juridico/PDFs/mesicic4_bra_sp.pdf
} 
descentralización, iniciativas destacadas como estrategias de Gestión efectiva de los recursos públicos. ${ }^{16}$

En el marco de la OPG, Paraguay presentó como iniciativa el Sistema de gestión de contratos y adquisiciones por catálogo electrónico y un Modelo estándar de control interno y México incluyó el Desarrollo de un Sistema Nacional de Fiscalización, así como un Nuevo Sistema de Contrataciones Públicas.

La mayoría de estas acciones se sustentan en perfeccionar los mecanismos de transparencia fiscal, contratación pública, evaluación de resultados, iniciativas de control fiscal y supervisión ciudadana del gasto público, haciendo más comprensible la información sobre ejecución presupuestaría, haciendo uso de componentes tecnológicos que fomenten el uso eficiente de los recursos y generen ahorro.

En el Estado de Sonora, el 06 de marzo de 2016 se implementó el portal tuobra.sonora.gob. $m x$ incluyendo información relativa a obra pública, licitaciones, transparencia y transmisión de eventos, que incluye un espacio para comentarios de la ciudadanía, relativos a la información que se presenta en este portal, cuyo objetivo es presentar en apego a transparencia, información sobre estos aspectos en forma explícita y clara, más allá de los requerimientos legales.

El portal tuobra.mx del Gobierno del Estado de Sonora, resultó ganador del primer lugar al Premio a la Innovación en Transparencia 2016, en la categoría Estatal, misma que está a cargo de la Subsecretaría de Desarrollo Administrativo y Tecnológico, adscrita a la Secretaría de la Contraloría General.

El objetivo de este portal es brindar la máxima transparencia y publicidad a los procesos de licitación, adjudicación y ejecución de obra pública, a través de un sistema de información abierto, dinámico y accesible por cualquier ciudadano desde cualquier computadora o teléfono inteligente. ${ }^{17}$

\footnotetext{
16 Vide: OGP (Open Government Pathership). 2012. "Country Commnitments". [en línea] Disponible en: http://www.opengovpartnership.org/conntries

17 Gobierno del estado de Sonora, "El portal TuObra.mx es una herramienta tecnológica diseñada para la rendición de cuentas y el fácil acceso del ciudadano a la información de las Licitaciones y Obra Pública en el Estado de Sonora.[en línea] disponible en: http://tuobra.sonora.gob.mx/
} 


\section{Gobierno abierto: Transparencia y acceso a la información en la administración de los recursos materiales}

Volumen 12, Número 22 ene-jun 2020

Dalia Navarro Olea

Ejemplos como este son una muestra del avance hacia la transparencia y la rendición de cuentas, que bajo el marco de Gobierno Abierto, proporciona la posibilidad de entablar conjuntamente, Ciudadanía y Administración Pública, la participación que se requiere para generar un mejor uso de los recursos públicos con el correspondiente cumplimiento de beneficio social.

\section{CONCLUSIONES Y RECOMENDACIONES}

La Administración de los Recursos Materiales ha tomado gran importancia por la gran cantidad de recursos financieros asignados a este rubro, situación que ha generado la necesidad de contar con servidores públicos altamente capacitados tanto en lo que respecta al marco legal como a la aplicación de procedimientos y apego a los valores institucionales, así como a la responsabilidad hacia la ciudadanía.

A nivel internacional la tendencia de Gobierno Abierto ha hecho inminente la necesidad de manejar información que permita que los ciudadanos cuenten con información sobre el fin de los recursos públicos en forma transparente, proporcionando datos que despejen dudas y sin ambigüedades.

El concepto de transparencia nunca había estado tan valorado en la historia no sólo de nuestro país sino particularmente en nuestro Estado, requiriendo con urgencia recobrar la credibilidad en las instituciones y promoviendo la participación de la población, no sólo como observadores silenciosos, sino como analistas participativos.

Queda claro la necesidad de modificar los mecanismos de transparencia en materia de contrataciones, obras públicas, licitaciones y compras directas, entre muchos otros aspectos relacionados al gasto público, situación que va en camino a la modernización derivada de la implementación de la estrategia de Gobierno abierto.

La experiencia de la transparencia focalizada en países de Latinoamérica proporciona bases para realizar ejercicios similares en la función pública en nuestra 
Administración de Recursos Materiales visión que puede verse aplicada con la reciente utilización de la página web tuobra,sonora.gob.mx, que seguramente se continuará desarrollando adicionando cada vez más información clara y útil, que permita la participación de la ciudadanía, proveedores y medios de comunicación, así como una mayor conectividad con las redes sociales.

Por último queremos incluir en este análisis la importancias de la selección del recurso humano en las áreas de compras, ya que como vimos es este un terreno fértil para la corrupción y que debe ser altamente valorado con parámetros de honestidad y responsabilidad, incluyendo personas capacitadas en diversas esferas del conocimiento, lo que posibilita una visión amplia de los problemas a los que se hará frente.

De mayor importancia y en forma especial, encontramos una marcada diferencia relacionada con el éxito en la implementación de estrategias de Gobierno y Transparencia basadas en el liderazgo, representado por el apoyo que se otorgue a la asignación de recursos para este rubro dependiendo de la prioridad que se le da a este aspecto en el Plan de Gobierno.

Sin embargo, la participación ciudadana no es automática, deberá recuperarse la legitimidad del gobierno involucrando paulatinamente a la población, empresarios, sindicatos, servidores públicos y todos los actores involucrados para que la misión de la administración pública cumpla con su objetivo de llevar a cabo el beneficio social para el que fue creado.

\section{REFERENCIAS BIBLIOGRÁFICAS}

ENRIQUEZ RUBIO Ernesto. Administración de recursos materiales en el sector público:Enfoque introductorio.pdf, [en línea]Disponible en : https://www.inap.mx/portal/images/pdf/book/14452.pdf

GOBIERNO ABIERTO: hacia un nuevo paradigma de gestión pública Red de Gobierno Electrónico de América Latina y el Caribe - Red GEALC Septiembre de 2013 Colección de documentos de trabajo sobre e-Gobierno 5 Oscar Oszlak.

https://www.oas.org/es/sap/dgpe/pub/coleccion5RG.pdf 


\section{Gobierno abierto: Transparencia y acceso a la información en la administración de los recursos materiales}

Volumen 12, Número 22 ene-jun 2020

Dalia Navarro Olea

GOBIERNO ABIERTO Y TRANSPARENCIA FOCALIZADA, Nicolás Dassen y Juan Cruz Vieyra Editores Noviembre 2012 / Código de publicaciones: IDBMG-137 Tendencias y desafíos para América Latina y el Caribe http://siare.clad.org/fulltext/2231500.pdf

GOBIERNO DEL ESTADO DE SONORA, Tu obra [en línea] Disponible en: http://tuobra.sonora.gob.mx/

LEY NÚMERO 90 el H. Congreso del Estado Libre y Soberano de Sonora, en nombre del pueblo, tiene a bien expedir la siguiente: Ley de Transparencia y Acceso a la Información Pública del Estado de Sonora, capítulo primero disposiciones generales http://www.stjsonora.gob.mx/acceso_informacion/marco_normativo/LEY\%2 ODE\%20TRANSPARENCIA\%20SONORA\%20NUEEVA.pdf

\section{NOTICIAS desde diferentes portales:}

GARIBAY ESCOBAR SERGIO, Debe tener la administración pública una gestión eficiente de los recursos'25 de Mayo de 2016, http://www.unison.edu.mx/noticias/default.php?id=21806

OGP (open Government Pathership). 2011. "Declaración sobre gobierno abierto". http://www.opengovpartnership.org/delcaración-sobre-gobiernoabierto

OGP (open Government Pathership). 2012. "Country Commnitments". http://www.opengovpartnership.org/conntries

RESTREPO HUNG Marcela, Promoción de la transparencia focalizada en la gestión del acceso a la vivienda en Colombia: el caso del programa TACS,. http://bibliotecadigital.tamaulipas.gob.mx/archivos/descargas/827d5b7339e1 d882b2fdd009d5c7a8d0c8617096.pdf

"OPEN GOVERNMENT: Collaboration, Transparency, and Participation in Practice. 2010. Don Tapscott (Lathrop y Ruma, 2010: XVI) Lathrop, D. y L. Ruma (eds.). Sebastopol: O'Reilly Media

SECRETARÍA DE LA CONTRALORÍA GENERAL DEL ESTADO DE SONORA “EI portal tuobra.mx gana el Premio a la Innovación en Transparencia 2016"

9 septiembre 2016..http://www.expreso.com.mx/del-editor/del-editor/142190-elportal-tuobramx-gana-el-premio-a-la-innovacion-en-transparencia-2016.html

Recibido 15 de abril de 2020

Aceptado 12 de mayo de 2020 